\title{
Japan Proton Accelerator Research Complex - J-PARC -
}

\author{
Shin'ya Sawada \\ High Energy Accelerator Research Organization (KEK), \\ 1-1 Oho, Tsukuba, Ibaraki 305-0801, Japan
}

\begin{abstract}
Japan Hadron Accelerator Research Complex, J-PARC, is a high-intensity proton accelerator complex now being built in Japan. The accelerator complex consists of a linac, a $3-\mathrm{GeV}$ and a $50-\mathrm{GeV}$ synchrotrons. The sciences to be conducted there, as well as characteristics of the facilities, are introduced, with the emphasis on nuclear and particle physics at the $50-\mathrm{GeV}$ facility.
\end{abstract}

\section{Overview}

Japan Proton Accelerator Research Complex [1], J-PARC, is a high-intensity proton accelerator complex now being built in Japan, which aims to extend the horizons of hadron beam sciences in the 21st century. The accelerator complex consists of a linac (linear accelerator), a $3-\mathrm{GeV}$ rapid cycling synchrotron, a $50-\mathrm{GeV}$ proton synchrotron, and several experimental facilities. A schematic view is shown in Fig. 1. The linac is the injector to the $3-\mathrm{GeV}$ synchrotron and will be used for $\mathrm{R} \& \mathrm{D}$ of nuclear transmutation systems in the future. The 3-GeV synchrotron delivers beams to the Materials and Life Science Facility (MLF), and works also as an injector to the 50-GeV synchrotron. The 50-GeV synchrotron delivers beams to the Hadron (HD) experimental hall and to a neutrino beam line. J-PARC is a joint project of the High Energy Accelerator Research Organization (KEK) and the Japan Atomic Energy Research Institute (JAERI). The facility is located in the Tokai site of JAERI on the Pacific Ocean coast in the neighborhood of existing research nuclear reactors. The site is about $70-\mathrm{km}$ northeast of KEK, and about $150-\mathrm{km}$ northeast of central Tokyo.

J-PARC is designed to be an intensity frontier facility. As can be seen in Fig. 2, the present world's hadron machines have typically 0.1-MW level of power both for low-energy (for materials sciences) accelerators and high-energy accelerators (high-energy physics) with one exception of the PSI cyclotron. The $3-\mathrm{GeV}$ and $50-\mathrm{GeV}$ synchrotrons will attain a ten times higher level of intensity of $1 \mathrm{MW}$, which is conpetitive to the SNS facility also now under construction in the United States. 


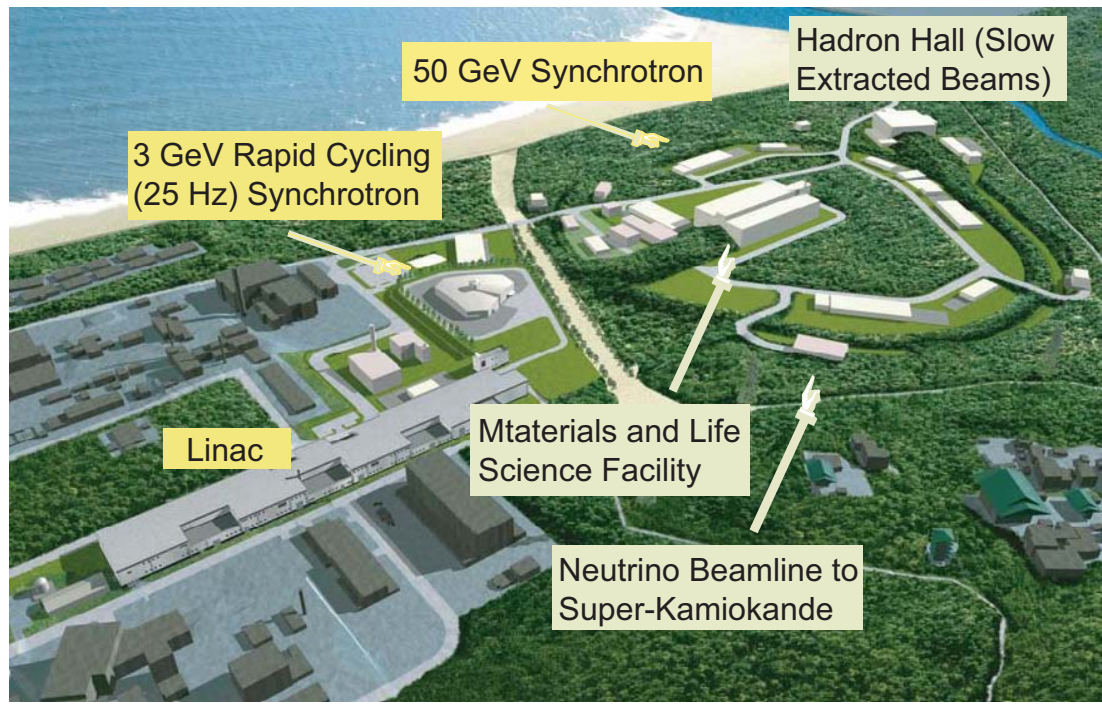

Figure 1: Schematic view of the J-PARC facility.

J-PARC has three major scientic goals: materials and life sciences at the $3-\mathrm{GeV}$ synchrotron facility, nuclear and high energy physics at the $50-\mathrm{GeV}$ synchrotron facility, and $\mathrm{R} \& \mathrm{D}$ toward nuclear transformation at the linac. The beams from the $3-\mathrm{GeV}$ synchrotron with 1-MW power will be used for a spallation neutron source and muon beams for materials and life sciences, by means of neutron diffraction/scattering/radiography and muon spin rotation $(\mu \mathrm{SR})$ techniques, respectively. The $50-\mathrm{GeV}$ synchrotron beam with a $15-\mu \mathrm{A}$ current, corresponding to 0.75-MW power, will lend high fluxes to secondary beams such as kaons, pions, and muons for nuclear and particle physics. A neutrino beam will be also one of the most important beams to do a long-baseline oscillation experiment. R\&D for accelerator-driven nuclear transmutation (ADS) will be conducted at a test experimental facility to be built in the future.

The Phase 1 of the J-PARC construction, which includes most of the accelerators and a part of the experimental facilities, started in 2001. Around the year of 2008 when the Phase- 1 construction is expected to be completed, there will be the $200-\mathrm{MeV}$ linac, the $3-\mathrm{GeV}$ synchrotron, the $50-\mathrm{GeV}$ synchrotron with 30 - or $40-\mathrm{GeV}$ operation, the MLF with several beam lines, the HD hall with possibly a few secondary beam lines, and the neutrino beam line (Fig. 3). We hope the Phase 2, which is the rest of the entire J-PARC project, will be approved soon after the completion of the Phase-1 construction.

In this talk, programs of nuclear and high-energy physics will be introduced, as well as a brief summary of materials and life sciences and ADS. The present status of the construction will be also presented. 


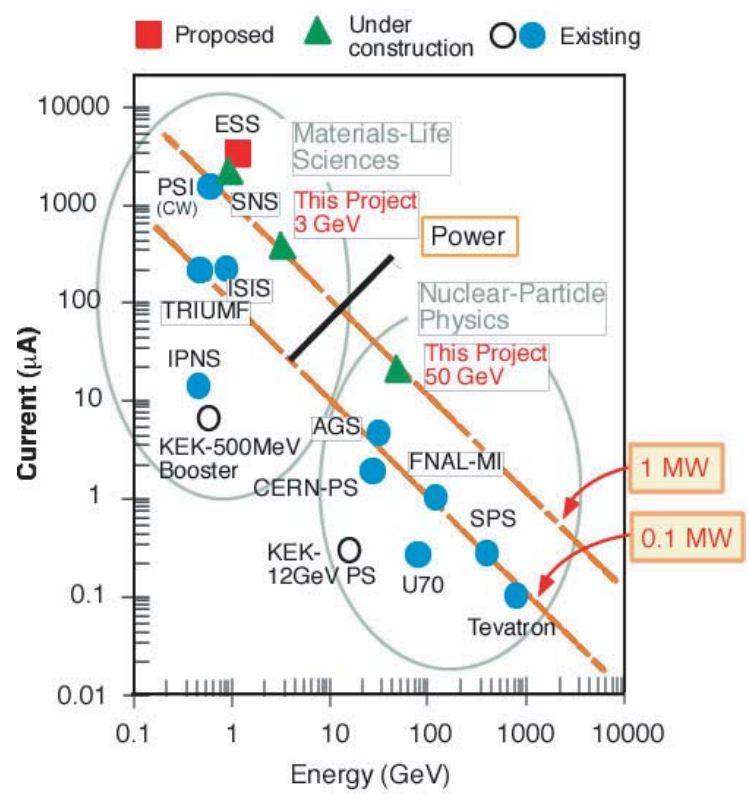

Figure 2: World hadron accelerators and their beam power. The product of the beam current (vertical axis) and the energy (horizontal axis) is the beam power. JPARC aims to be at the frontier of beam power.

\section{Nuclear and particle physics at J-PARC}

The beams from the $50-\mathrm{GeV}$ synchrotron will be used for nuclear and particle physics by means of secondary as well as primary beams. There will be two extracted beams from the $50-\mathrm{GeV}$ synchrotron: one is the slow extracted beam used for fixed target experiments at the HD hall, and the other is the fast extracted beam for neutrino beam production.

The ideas of experiments which use these beams have been widely discussed. A series of the workshops has been held to develop discussions of the physics cases, such as the International Workshops on Nuclear and Particle Physics at J-PARC (NP01, NP02, and NP04) [2]. Letters of Intent (LoI's) for nuclear and particle physics experiments [3] were called in July of 2002, and 30 LoI's were collected by the beginning of 2003. Among these, 6 LoI's are on strangeness nuclear physics, 7 on nuclear/hadron physics, 4 on kaon decay physics, 3 on muon physics, 1 on neutrino physics, and 9 on future facilities. The total number of the authors is 478, with almost equal members from among Japan, Europe, and the U.S.

In the following, the neutrino experiment using the fast extracted beam is introduced first, then examples of the experiments at the hadron hall, hypernuclear spectroscopy, hadron physics experiments, and kaon rare decay experiment are presented. Of course, 


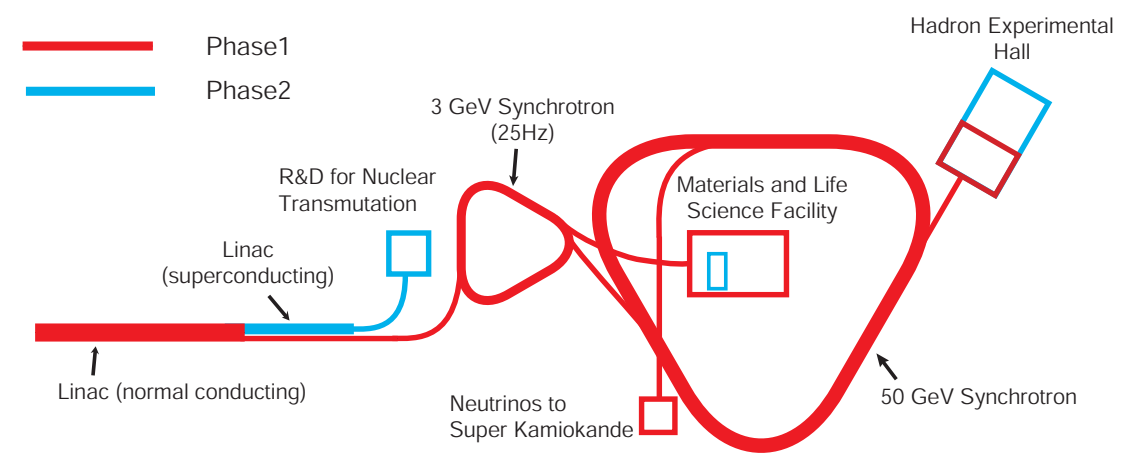

Figure 3: Phase 1 and Phase 2 of J-PARC. The major parts of the accelerators and some of the experimental facilities are included in Phase 1. The Phase-1 construction started in 2001, and is expected to be completed around 2008.

details of the experimental programs will be determined after an official recommendation by the PAC (Physics/Program Advisory Committee), but these physics topics are strong candidates to be pursued at the $50-\mathrm{GeV}$ facility of J-PARC.

\subsection{Neutrino oscillation experiment}

A long-baseline neutrino oscillation experiment is one of the most attracting topics at J-PARC [4]. Muon neutrinos $\left(\nu_{\mu}\right)$ are produced by proton beams from the $50-\mathrm{GeV}$ synchrotron, and launched to the Super-Kamiokande (SK) detector located at Kamioka, about $295 \mathrm{~km}$ west of the J-PARC site. Observations with atmospheric neutrinos, such as the SK, discovered neutrino oscillations and thus finite masses of neutrinos, and the K2K (KEK-to-Kamioka) experiment confirmed the oscillations with "man-made" neutrinos. With these backgrounds, there are three motivations of the oscillation experiment at J-PARC named T2K (Tokai-to-Kamioka):

- Precise measurement of disappearance $\nu_{\mu}$ to $\nu_{x}$,

- Discovery of $\nu_{e}$ appearance, and

- $\mathrm{CP}$ violation in the neutrino sector in the future with higher beam intensity and a new detector.

Please note that the intensity of $\nu_{\mu}$ around $1 \mathrm{GeV}$ will be about 100 times higher than the K2K experiment. In order to produce an optimum neutrino beam, a method named "offaxis beam" is used. The off-axis beam is a wide-band beam with a $\pi^{+}$beam intentionally misaligned from the detector axis. With this method, a quasi-moochromatic beam can be obtained with higher intensity than a narrow band beam. The beam energy is adjusted so that it matches to the energy at which the maximum oscillation occurs. 


\subsection{Experiments at the hadron (HD) hall}

Figure 4 shows the layout around the HD Hall. Proton beams extracted from the $50-\mathrm{GeV}$ synchrotron are conducted to the primary beam line at the switchyard. At the beginning of the Phase 1 (day-1), only one primary beam line (A) will be prepared. Also, the construction of the K1.8 secondary beam line has a higher priority at this time for the day-1 experiments. The buildings and the shields are designed so that other beam lines can be built even at a later time in the phase.

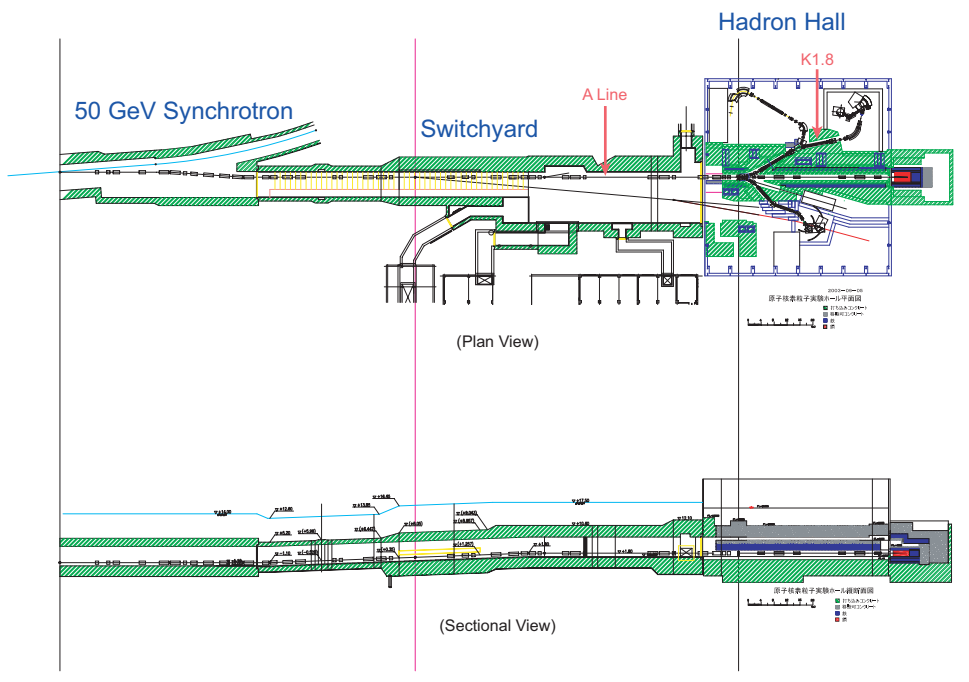

Figure 4: Layout around the Hadron Hall.

Hypernuclear spectroscopy One of the major physics topics of J-PARC is hypernuclear physics, which makes the best use of high intensity of secondary kaon/pion beams. We can imbed hyperons in deep nuclear matter, because hyperons are free from Pauli blocking, where we can investigate the medium effects. They are a major issue in present nuclear physics, through hyperon effective mass, potential, and hypron-nucleon interactions by means of hyperon spectroscopy.

The high-intensity $\mathrm{K}^{-}$beam at $\sim 1.8 \mathrm{GeV} / \mathrm{c}$ available at the $50-\mathrm{GeV}$ synchrotron is quite unique to open a new frontier of strangeness nuclear physics in the spectroscopic studies of $\mathrm{S}=-2$ systems, such as hypernuclei (no $\Xi$ hypernucleus has been found yet) and double- $\Lambda$ hypernuclei (only a few examples) [5]. This is not only a natural extension from the $\mathrm{S}=-1$ systems, but also a significant step to explore multi-strangeness hadronic systems. With the studies of $\mathrm{S}=-2$ systems, we can obtain ideas of the properties of strange hadronic (nuclear) matter in the core of a neutron star, for example. It is also important to extract information on the $\Xi$ single particle potential and $\Xi \mathrm{N}$ and $\Lambda \Lambda$ interactions from 
the spectroscopic data. Note that the knowledge of the depth of the $\Xi$-nucleus potential is important for estimating the existence of strange hadronic matter.

Experimentally, $\mathrm{S}=-2$ systems can be well studied with the $\left(K^{-}, K^{+}\right)$reactions through an elementary process, $K^{-}+p \rightarrow K^{+}+\Xi^{-}$. A $\mathrm{K}^{-}$beam with the best quality in the world will be supplied at the HD hall. For a $1.8 \mathrm{GeV} / \mathrm{c} \mathrm{K}^{-}$beam, the intensity will be $1 \times 10^{7}$ /sec obtained with good momentum resolution. The $\mathrm{K}^{-} / \pi^{-}$ratio will be more than 1 . The produced $\mathrm{K}^{+}$mesons will be analyzed by the existing SKS spectrometer, which has wide acceptance $(\sim 50 \mathrm{msr})$ and good momentum resolution $(\sim 2 \mathrm{MeV}$ (FWHM)). The yield of the production of $\Xi$ hypernuclei for a ${ }^{208} \mathrm{~Pb}$ target $\left(2 \mathrm{~g} / \mathrm{cm}^{2}\right)$ is estimated to be about 6 events per day. So, even for this heaviest case, we could get enough statistics within about 20 days to obtain spectroscopic information. For lighter targets, such as ${ }^{28} \mathrm{Si}$ and ${ }^{58} \mathrm{Ni}$, the yields are several times higher.

Hadron physics experiments Hadron physics is regarded to be physics on hadronic matter. The parton picture and QCD play a crucial role there, because quarks and gluons are the fundamental elements of hadronic matter and QCD is the fundamental theory to describe forces between them. While hadron physics covers a very wide range of fields, three topics are introduced below: kaonic nuclei, pentaquark systems, and sea quark distributions at large $\mathrm{x}_{\mathrm{Bj}}$.

Recently a $\mathrm{K}^{-}$ppn bound state was discovered [6]. The experiment was performed at the KEK-PS using the $K^{-}+{ }^{4} H e \rightarrow K^{-} p p n+n$ reaction, and the peak, which corresponds to the $\mathrm{K}^{-}$ppn bound state, was found in the neutron momentum distribution. According to a theoretical calculation [7], the density at the center of the system is much larger than the normal nuclei, such as ${ }^{3} \mathrm{He}(=\mathrm{ppn})$. This is really a new phenomenon, which opens a way to research of high density nuclear matter, and this kind of research should be continued at J-PARC also [8].

One of the hottest topics in hadron physics is the pentaquark systems. Though extensive efforts have been made after the discovery of a pentaquark system, $\Theta^{+}$, announced by the SPring-8/LEPS group [9], its production cross section, width, spin and parity are still unclear. Hadron reactions are important to study these properties of the $\Theta^{+}$. An experiment to measure $p\left(K^{+}, \Theta^{+} \pi^{+}\right) n$ reactions is being prepared at the KEK-PS, whose goal is to take data with much more statistics and determine the width of $\Theta^{+}$, the reaction cross section, and information on the spin and parity. At J-PARC, the phase shift measurement of the $K^{+}+n$ system would be a good candidate for an experiment. In addition, $\Theta^{+}$nuclei and other pentaquark systems can be studied. Charmed pentaquark systems formed by neutrino beams would also be interesting.

Another example of hadron physics experiments is a measurement of muon pairs from $\mathrm{p}+\mathrm{p}, \mathrm{p}+\mathrm{d}$, and $\mathrm{p}(\mathrm{d})+\mathrm{A}$ reactions. Its major motivation is to measure the flavor asymmetry of the sea quark distribution $(\bar{d} / \bar{u})$ at large $\mathrm{x}_{\mathrm{Bj}}$. This is an experiment to study the parton model and QCD in a direct way, and can have its uniqueness with the $50-\mathrm{GeV}$ proton beam [10].

Kaon rare decay experiments The motivation of the kaon rare decay experiments is to determine elements of the CKM matrix and confirmation of the unitary triangle. The most useful decays are the flavor changing neutral current decays of $K_{L} \rightarrow \pi^{0} \nu \bar{\nu}$ and 
$K^{+} \rightarrow \pi^{+} \nu \bar{\nu}$. The former is a direct CP-violating process dominated by short distance contributions. A current estimate from the Standard Model of the branching ratio for this process is $3 \times 10^{-11}$. A pilot experiment (KEK-PS E391A), aiming at a sensitivity of $10^{-9 \sim-10}$, is running at KEK, and an experiment named KOPIO, aiming at a $10^{-12}$ sensitivity, is in preparation at the BNL-AGS. The E391A setup, when moved to the $50-\mathrm{GeV}$ synchrotron, will achieve a sensitivity of $10^{-14}$ in a runtime of $3 \times 10^{7}$ seconds, accumulating 1000 events [11]. This sensitivity is more than the Standard Model prediction of about $3 \times 10^{-11}$.

\section{Other sciences at J-PARC}

At the MLF, high intensity pulsed neutrons will be produced, as well as low energy muons from a separated target. In materials and life sciences, neutron beams are very important and useful. The neutron carries two unique features. One is that the neutron does not have any electric charge, and has a mass which is close to the proton mass. Thus, neutrons are scattered by atomic nuclei and also by light-mass nuclei. This feature is distinct if one compares neutrons with X-rays. Because X-rays are scattered by atomic electrons, they can observe atoms with large atomic numbers. Thus neutrons can probe lighter atoms, such as oxygen, lithium (in a lithium battery, for example), and also $\mathrm{H}_{2} \mathrm{O}$ in a biological molecule, while X-rays can probe heavier atoms. The other significant feature of the neutron is that it carries a magnetic moment. The neutron is a microscopic magnet. Therefore, magnetic scattering of neutrons can reveal the microscopic magnetic structure. The sciences conducted at the muon facility at the MLF are to be covered by a contribution to this conference by Dr. N. Kawamura [12].

Another goal of J-PARC is to conduct R\&D for nuclear transmutation, which aims to reduce long-lived radioactive nuclei produced in nuclear fuel plants. R\&D experiments are planned to study various issues to realize the concept of accelerator-driven nuclear transmutation, such as radiation tests of materials etc.

\section{Construction schedule and status}

The Phase- 1 construction started in 2001. We expect the first beam to the experimental facilities in 2008. Figure 5 shows a recent aerial photograph of the construction site. Buildings of the linac and the $3-\mathrm{GeV}$ synchrotron are almost completed. Tunnels and buildings of the 50-GeV synchrotron, the MLF, the HD hall, and the neutrino beam line are extensively under construction. Many of the accelerator components, such as the $\mathrm{H}^{-}$ ion source, the RFQ, a part of the drift tube lina, and the magnets, have already been manufactured and are being tested. $\mathrm{R} \& \mathrm{D}$ for the beam lines and detectors, including design, mock-ups, tests etc., is being done, and we are almost ready to manufacture and install them to the experimental facilities, waiting for the building completion. 


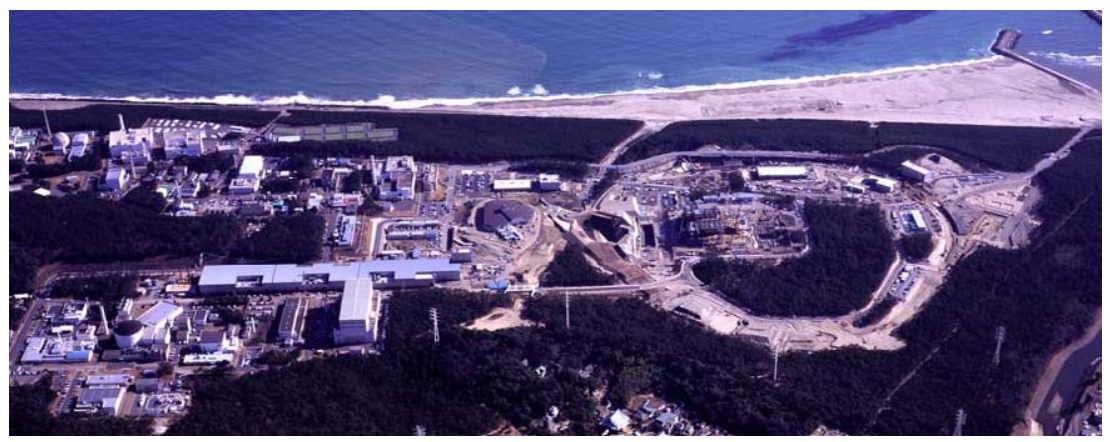

Figure 5: Photograph of the construction site taken in January, 2005.

\section{Summary}

J-PARC will be the highest intensity accelerator complex of the $\mathrm{GeV}$ and ten-GeV energy regions in the world. The major aims are materials and life sciences by the $3-\mathrm{GeV}$ synchrotron, nuclear and particle physics by the $50-\mathrm{GeV}$ synchrotron, and R\&D for nuclear transmutation technology by the linear accelerator. The Phase-1 construction began 2001, and will be completed in 2008. There is a wide variety of physics possibilities. Participation from all over the world is welcome!

\section{References}

[1] http://j-parc.jp/ .

[2] NP01: http://www-ps.kek.jp/jhf-np/NP01/ , NP02: http://www-ps.kek.jp/jhfnp/NP02/, NP04: http://j-parc.jp/NP04/ .

[3] The LoI's are listed at http://www-ps.kek.jp/jhf-np/LOIlist/LOIlist.html .

[4] Information of the neutrino experiment at J-PARC can be found at http://neutrino.kek.jp/jhfnu/ .

[5] K. Imai et al., L06: New Generation Spectroscopy of Hadron Many-Body Systems with Strangeness $\mathrm{S}=-2$ and $-1,[3]$.

[6] M. Iwasaki et al., nucl-ex/0310018.

[7] A. Dote et al., Phys. Rev. C 70 (2004) 044313.

[8] T. Nagae et al., L10: Study of Dense Kbar Nuclear Systems, [3].

[9] T. Nakano et al., Phys. Rev. Lett. 91 (2003) 012002.

[10] S. Sawada et al., L15: Physics of High-mass Dimuon Production at 50-GeV Proton Synchrotron, [3].

[11] T. Inagaki et al., L05: Measurement of the $K_{L}^{0} \rightarrow \pi^{0} \nu \bar{\nu}$ Branching Ratio, [3].

[12] N. Kawamura, in these proceedings. 